\title{
Collision Avoidance of 3D Rectangular Planes by Multiple Cooperating Autonomous Agents
}

\author{
Jai Raj, ${ }^{1}$ Krishna Raghuwaiya $\mathbb{D}^{2}{ }^{2}$ and Jito Vanualailai ${ }^{1}$ \\ ${ }^{1}$ School of Computing Information \& Mathematical Sciences, The University of the South Pacific, Suva, Fiji \\ ${ }^{2}$ School of Education, The University of the South Pacific, Suva, Fiji \\ Correspondence should be addressed to Krishna Raghuwaiya; raghuwaiya_k@usp.ac.fj
}

Received 14 February 2020; Revised 23 September 2020; Accepted 8 October 2020; Published 28 October 2020

Academic Editor: Wenqing Wu

Copyright (C) 2020 Jai Raj et al. This is an open access article distributed under the Creative Commons Attribution License, which permits unrestricted use, distribution, and reproduction in any medium, provided the original work is properly cited.

We develop a set of novel autonomous controllers for multiple point-mass robots or agents in the presence of wall-like rectangular planes in three-dimensional space. To the authors' knowledge, this is the first time that such a set of controllers for the avoidance of rectangular planes has been derived from a single attractive and repulsive potential function that satisfies the conditions of the Direct Method of Lyapunov. The potential or Lyapunov function also proves the stability of the system of the first-order ordinary differential equations governing the motion of the multiple agents as they traverse the three-dimensional space from an initial position to a target that is the equilibrium point of the system. The avoidance of the walls is via an approach called the Minimum Distance Technique that enables a point-mass agent to avoid the wall from the shortest distance away at every unit time. Computer simulations of the proposed Lyapunov-based controllers for the multiple point-mass agents navigating in a common workspace are presented to illustrate the effectiveness of the controllers. Simulations include towers and walls of tunnels as obstacles. In the simulations, the point-mass agents also show typical swarming behaviors such as split-and-rejoin maneuvers when confronted with multiple tower-like structures. The successful illustration of the effectiveness of the controllers opens a fertile area of research in the development and implementation of such controllers for Unmanned Aerial Vehicles such as quadrotors.

\section{Introduction}

The motion planning and control (MPC) of mobile robots or agents is a challenging task and an interesting problem attracting considerable attention to the robotic community over the last couple of decades. The design of a particular robotic system and motion planning are usually treated independently [1]. Typically, MPC algorithms are applied to systems with fully fixed geometric and kinematic features, while the system design in robotics takes into account robustness, stiffness, workspace volume, obstacle avoidance schemes, and other performance features. The principle goal for any MPC problem is to find the most optimum design to optimise the motion between given configurations [2-7]. In an MPC problem, multiple robots are favoured as they are able to cooperate for faster and more efficient results $[4,5,8-10]$, including other fields where multiagent operations are always preferred [11].
Path planning or MPC algorithms for mobile robots operating in an environment cluttered with obstacles are usually grouped according to the methodologies used to generate the geometric path, namely, the road map techniques, cell decomposition algorithms, and artificial potential field (APF) methods $[4,12]$. These path planning algorithms have a common objective, which is to find the shortest and most optimal geometric path taking into account the moving objects and obstacles in the workspace [13-15]. While the calculation of a hindrance-free way may take care of numerous significant issues in industrial settings where the robot may move cautiously, it is inadequate and practically futile when the robot needs to move at sensibly high speeds, for example, multiple mobile robots navigating through dynamic cluttered situations and autonomous vehicles navigating in a highway traffic situation.

In this research article, we use the Lyapunov controllers, constructed via the Lyapunov-based Control Scheme 
(LbCS), essentially an APF method, for the control and stability of a system point-mass mobile robots that, in theory, can take on reasonably high velocities. The LbCS has been employed to warrant point and posture stabilities in the sense of Lyapunov for MPC for various robotic systems, such as car-like mobile robotic systems [4], mobile manipulators [16], tractor-trailer systems [12, 17], and swarming [18]. We utilise the control scheme to derive and extract centralised velocity-based control laws for pointmass mobile robots.

1.1. Contributions. The novelty of this paper is the ease in developing autonomous controllers for the avoidance of three-dimensional wall-like rectangular planes by a mobile robot or agent while it is in motion using a technique known as the Minimum Distance Technique (MDT). The ability to do this opens up many possibilities. Walls can be used to model buildings and towers, windows, and doors. They can be used to model highways and tunnels. When we deal, for instance, with autonomous Unmanned Aerial Vehicles (UAVs), it is now possible to model a drone's performance in the face of such obstacles as buildings and tunnel walls, and its maneuverability inside buildings clustered with rectangular objects and exited. For disaster surveillance and in an urban war simulation and situation, this maneuverability is critical $[19,20]$.

The MDT was introduced by Sharma et al. [21] to create parking bays for the posture control problem of robotic systems and avoid the sides of a bay, modelled as straight lines. The MDT uses APF functions for the avoidance of the boundaries of the parking bay. In this paper, we extend the methodology to encompass rectangular planes. The MDT involves the computation of the minimum distance from the centre of the point-mass mobile robot to the surface of the rectangular plane and the avoidance of the resultant point on the st $\geq 0$ urface of the rectangular plane. The avoidance of the nearest point on the surface of the rectangular plane at any time $t \geq 0$ ensures that the point-mass mobile robot avoids the whole plane. As we shall see, this algorithm helps in simplifying the navigation laws. Surely, there are other methods of obstacle avoidance of polygons. The most recent one was proposed by Arantes et al. [22] who discussed path planning approaches for dynamic systems to handle nonconvex constraints to be formulated as model-predictive control, which planned discrete time control and state sequences simultaneously through a constrained optimisation. The optimisation problem that needs to be solved in this case is the mixed-integer linear programming (MILP) when the dynamics are linear and the obstacles are represented by combinations of polytopes, with no uncertainty presence. The problem that lies in this particular approach is the jumps between the time steps, which could result in a trajectory cutting through the obstacle, given that the method is only concerned with satisfying the constraints at a discrete point in times, as shown in Figure 1(a). Arantes et al. devised a new approach to suppress this problem by imposing constraints that require every pair of adjacent states to be on the same side of an obstacle, as shown in Figure 1(b) [23].

Furthermore, comparing Arantes et al. approach to the MDT, the latter results in a smooth, continuous path for the avoidance of irregular shaped (rectangular plane) obstacles. An illustration of the MDT for the avoidance of a rectangular plane is shown in Figure 1(c).

The main contributions of this paper are summarised as follows:

(1) The design of the velocity algorithm for a point-mass mobile robot which is based on a Lyapunov function that acts as an energy function of the system. The velocity algorithm ensures safe, collision-free trajectories that converge to the intended target.

(2) The design of the velocity algorithm for the pointmass mobile robot which is based on the development of a Lyapunov function that acts as an energy function of the system. The velocity algorithm applied here is altogether not quite the same as the ones in the literature. Consistently enduring velocities are utilised; nonetheless, the robot needs to stop after it has accomplished its objective. This stop should not be unexpected by a truncation of speed; rather, the robot should slow down its motion and afterward come to rest. The velocity algorithm and the objective target intended for the robot guarantee a protected and safe stop at the goal objective and furthermore guarantee that the robot stays there.

(3) A three-dimensional rectangular-plane obstacle avoidance scheme using the MDT. While in motion, the distance between the point-mass robot and the closest point on the surface of the wall is computed and the point-mass robot avoids this point on the surface of the wall, resulting in the avoidance of the entire wall. In addition, we only consider the wall closest to the point-mass robot en route to its target. Subsequently, our obstacle avoidance scheme is more straightforward contrasted with, for instance, the avoidance schemes used in the artificial potential strategies where all of the obstacles are considered in parallel $[4,17]$.

(4) Stability analysis pertaining to the kinodynamic system. We use the Direct Method of Lyapunov to carry out the stability analysis, proving that the equilibrium point of the system, representing the target of a point mass, is stable.

The paper is organised as follows: In Section 2, we define the kinematic model of the point-mass robot; in Section 3, the APF functions are defined; in Section 4, the Lyapunov function is constructed and the robust nonlinear continuous control laws for the mobile robot are extracted; in Section 5, the stability of the system is discussed; in Section 6, the simulation results are presented to show the robustness and effectiveness of the proposed control inputs and followed by conclusion in Section 7. 


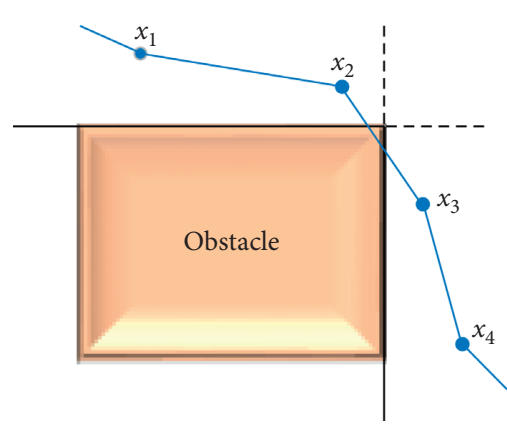

(a)

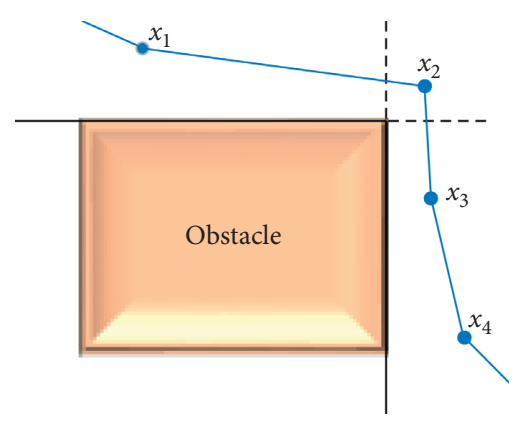

(b)

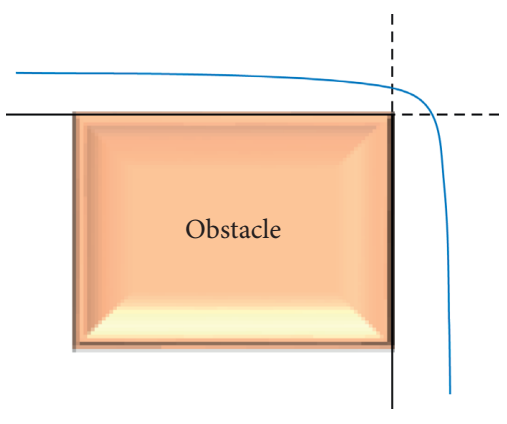

(c)

FIGURE 1: (a) Conventional model-predictive control-based path planning requires that states are outside of polygonal obstacles resulting in the path cutting through the obstacle. (b) The MILP approach of using a straight-line trajectory between waypoints [22]. (c) The smooth and continuous path for MDT demonstration of obstacle avoidance.

\section{Modelling a Point-Mass Robot or Agent in 3D}

The modelling process of a robotic system involves the conceptualisation of the problem, residing on the abstraction level. Simulation, however, mainly focuses on the implementation of the execution of the model to study the behavior and performance of an actual or theoretical system. This section proposes a simple kinematic model for the moving point-mass robot, an abstraction of a simple form of a robotic system. A two-dimensional schematic representation of a point-mass robot with and without rectangular obstacle avoidance is shown in Figure 2. We begin with the following definition.

Definition 1. A point mass, $\mathscr{P}_{i}$, is a sphere of radius $\mathrm{rp}_{i}$ and centred at $\left(x_{i}(t), y_{i}(t), z_{i}(t)\right) \in \mathbb{R}^{3}$ for $t \geq 0$. That is, it is the set

$$
\mathscr{P}_{i}=\left\{\left(Z_{1}, Z_{2}, Z_{3}\right) \in \mathbb{R}^{3}:\left(Z_{1}-x_{i}\right)^{2}+\left(Z_{2}-y_{i}\right)^{2}+\left(Z_{3}-z_{i}\right) \leq \mathrm{rp}_{i}^{2}\right\} .
$$

At time $t \geq 0$, the instantaneous velocity of the point mass will be given as $\left(v_{i}(t), w_{i}(t), u_{i}(t)\right)=\left(\dot{x}_{i}(t), \dot{y}_{i}\right.$ $\left.(t), \dot{z}_{i}(t)\right)$. Assuming the initial conditions, a system of the first-order ODEs governing $\mathscr{P}_{i}$ is

$$
\left.\begin{array}{l}
\dot{x}_{i}=v_{i}(t), \dot{y}_{i}=w_{i}(t), \dot{z}_{i}=u_{i}(t), \\
x_{i 0}:=x_{i}\left(t_{0}\right), y_{i 0}:=y_{i}\left(t_{0}\right), z_{i 0}:=z_{i}\left(t_{0}\right),
\end{array}\right\}
$$

for $\quad i=1, \ldots, n$. Let $\quad \mathbf{x}_{i}:=\left(x_{i}, y_{i}, z_{i}\right) \in \mathbb{R}^{3} \quad$ and $\mathbf{x}:=\left(\mathbf{x}_{1}, \ldots, \mathbf{x}_{n}\right) \in \mathbb{R}^{3 n}$.

Next, we will formulate the components that form the Lyapunov function, essentially the attractive and repulsive potential field functions.

\section{Construction of the APF Functions}

In this section, we construct the components of the Lyapunov function. We assume that $\mathscr{P}_{i}$ has a priori knowledge of the entire workspace. The principle objective is to construct the Lyapunov function from which we derive the nonlinear velocity control inputs $v_{i}(t), w_{i}(t)$, and $u_{i}(t)$ for $i=1, \ldots, n$ such that $\mathscr{P}_{i}$ navigates and reaches its target configuration, avoiding any obstacle, whether fixed, moving, or artificial, while it is in motion. The design of the nonlinear control inputs is captured in Figure 3, clearly illustrating the roles of the individual components in the design of the control scheme.

3.1. Attractive Potential Field Functions. We introduce basic mathematical notions to design and construct attractive functions for target attraction for $\mathscr{P}_{i}$.

3.1.1. Attraction to Target Function. To initiate movement and ensure convergence, we propose to have a target $T_{i}$ for each of the point-mass mobile robots $\mathscr{P}_{i}$. The convergence of $\mathscr{P}_{i}$ to $T_{i}$ will be guaranteed by the Lyapunov function.

Definition 2. The assigned target for the point-mass mobile robot of $\mathscr{P}_{i}$ is a sphere with centre $\left(\tau_{i 1}, \tau_{i 2}, \tau_{i 3}\right)$ and radius $r \tau_{i}$. That is, it is the set

$$
T_{i}=\left\{\left(Z_{1}, Z_{2}, Z_{3}\right) \in \mathbb{R}^{3}:\left(Z_{1}-\tau_{i 1}\right)^{2}+\left(Z_{2}-\tau_{i 2}\right)^{2}+\left(Z_{3}-\tau_{i 3}\right)^{2} \leq r \tau_{i}^{2}\right\} .
$$

The next function will measure the Euclidean distance of $\mathscr{P}_{i}$ from its designated target $T_{i}$ at time $t \geq 0$. It will be used as an attraction function:

$$
V_{i}(\mathbf{x})=\frac{1}{2}\left[\left(x_{i}-\tau_{i 1}\right)^{2}+\left(y_{i}-\tau_{i 2}\right)^{2}+\left(z_{i}-\tau_{i 3}\right)^{2}\right] .
$$

An illustration of the total potentials for the target attraction function is shown in Figure 4(a), while Figure 4(b) shows the analogous contour plot generated over a workspace $0<Z_{1}<100$ and $0<Z_{2}<100$ for the point-mass mobile robot. For simplicity, we consider the target function in a 2-dimensional environment. The disk-shaped target for the point-mass mobile is fixed at $\left(\tau_{01}, \tau_{02}\right)=(50,50)$ with a radius of $\mathrm{rp}_{0}=1$.

3.1.2. Auxiliary Function. In the MPC problem, $\mathscr{P}_{i}$ starts from an initial position and navigates towards its target. While navigating, the motion of $\mathscr{P}_{i}$ is such that it will avoid 


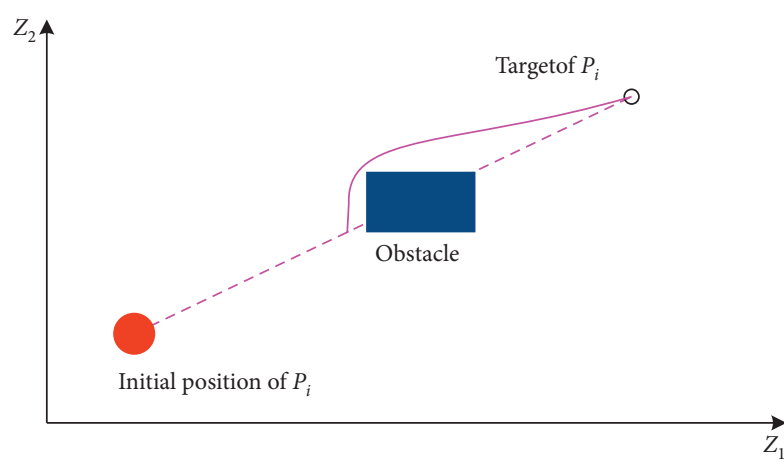

FIgURE 2: $2 \mathrm{D}$ schematic representation of $\mathscr{P}_{i}$ with planar obstacle in $Z_{1}-Z_{2}$ plane.

all obstacles, whether it is fixed or moving, with respect to the kinodynamic constraints that are tagged with the robotic system including the constraints on velocity and angles before reaching its objective target. Once it has reached the target, it essentially means that it has accomplished the task that was given to the robot, and hence it needs to stop at the target configuration. Intuitively, this means that the energy of the robotic system needs to be zero at the target configuration; that is, the nonlinear controllers need to vanish at the target. Hence, to achieve this and to ensure the convergence of $\mathscr{P}_{i}$ to its target configuration, we consider the auxiliary function of the form

$$
G_{i}(\mathbf{x})=V_{i}(\mathbf{x}) \text {. }
$$

\subsection{Repulsive Potential Field Functions}

3.2.1. Workspace Boundaries. We shall confine the motion of $\mathscr{P}_{i}$ in a cuboid constrained by the dimensions $\eta_{1} \times \eta_{2} \times \eta_{3}$. Since the motion is confined within these boundary walls, the walls are hence treated as fixed obstacles. Therefore, for the avoidance of these walls, the following functions are proposed:

$$
\left.\begin{array}{l}
\mathrm{WS}_{i 1}(\mathbf{x})=x_{i}-\mathrm{rp}_{i}, \mathrm{WS}_{i 2}(\mathbf{x})=\eta_{2}-\left(y_{i}+\mathrm{rp}_{i}\right), \\
\mathrm{WS}_{i 3}(\mathbf{x})=\eta_{1}-\left(x_{i}+\mathrm{rp}_{i}\right), \mathrm{WS}_{i 4}(\mathbf{x})=y_{i}-\mathrm{rp}_{i}, \\
\mathrm{WS}_{i 5}(\mathbf{x})=z_{i}-\mathrm{rp}_{i}, \mathrm{WS}_{i 6}(\mathbf{x})=\eta_{3}-\left(z_{i}+\mathrm{rp}_{i}\right),
\end{array}\right\}
$$

for $i=1, \ldots, n$, which are positive within the rectangular cuboid.

3.2.2. Rectangular-Plane Obstacle Avoidance. Disks in $2 \mathrm{D}$ and spheres in 3D are the simplest models of obstacles. However, they encompass extraspaces that are not needed for avoidance. For example, enclosing a rod-like structure within a sphere introduces spaces that need not be avoided. As an illustration, Figure 5(a) shows the contour plot of the total potentials and the corresponding collision-free path of a point mass over the workspace $0<Z_{1}<40$ and $0<Z_{2}<40$ encompassing a rod-shaped obstacle, while Figure 5(b) showcases the contour plot of the total potentials and the resulting path if the rod is

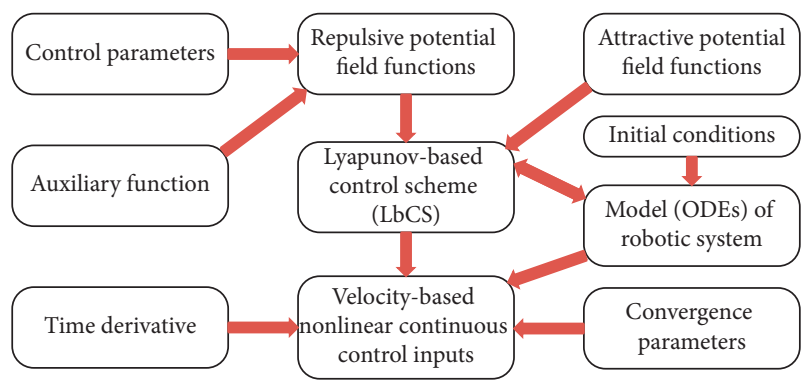

Figure 3: The block diagram outlining the LbCS design.

replaced by a disk-shaped obstacle. The initial and final coordinates of the rod are $(10,20)$ and $(30,20)$, respectively. The disk portrayal of the rod has a diameter of 10 , which matches the length of the rod, and is centred at $(20,20)$. The path generated in the presence of the rodshaped obstacle is optimal in terms of the distance traversed since the obstacle space is small in contrast to the disk portrayal of the rod. Therefore, in this article, we introduce rectangular obstacles.

To avoid the rectangular obstacles via the MDT, the surface wall of the rectangular plane is classified as a fixed obstacle. Let us fix $\ell=1, \ldots, \bar{m}, \bar{m} \in \mathbb{N}$ rectangular-planeshaped obstacles within the workspace. An illustration of a rectangular plane is showcased in Figure 6, which we shall use to derive the new mathematical equations for its avoidance. Three points are sufficient for deriving the saturation functions and hence designing the rectangularplane avoidance functions. We begin with the following definition.

Definition 3. Assume that the three-dimensional $\ell$ th planar obstacle has the three coordinates $\left(a_{\ell 1}, b_{\ell 1}, c_{\ell 1}\right)$, $\left(a_{\ell 2}, b_{\ell 2}, c_{\ell 2}\right)$, and $\left(a_{\ell 3}, b_{\ell 3}, c_{\ell 3}\right), \ell=1, \ldots, \bar{m}, \bar{m} \in \mathbf{N}$ (see Figure 6). A single point in the plane is defined by

$$
\mathrm{SP}_{i \ell}:=\left(Z_{1}, Z_{2}, Z_{3}\right):=\left(P x_{i \ell}, P y_{i \ell}, P z_{i \ell}\right) \in \mathbb{R}^{3} \text {. }
$$

Then the plane can be precisely described by the set

$$
P_{\ell}=\left(\mathrm{SP}_{1 \ell}, \mathrm{SP}_{2 \ell}, \ldots, \mathrm{SP}_{n \ell}\right) \in \mathbb{R}^{3 n} \text {. }
$$

Then the set of $\ell$ planes, $\ell \in \bar{m}$, is

$$
\mathrm{CP}=\left\{P_{1}, P_{2}, \ldots, P_{\bar{m}}\right\} \in R^{3 n \bar{m}},
$$

where $P x_{i \ell}=a_{\ell 1}+\lambda_{i \ell 1}\left(a_{\ell 2}-a_{\ell 1}\right)+\lambda_{i \ell 2}\left(a_{\ell 3}-a_{\ell 1}\right), \quad P y_{i \ell}=$ $b_{\ell 1}+\lambda_{i \ell 1}\left(b_{\ell 2}-b_{\ell 1}\right)+\lambda_{i \ell 2}\left(b_{\ell 3}-b_{\ell 1}\right)$, and $P z_{i \ell}=c_{\ell 1}+\lambda_{i \ell 1}$ $\left(c_{\ell 2}-c_{\ell 1}\right)+\lambda_{i \ell 2}\left(c_{\ell 3}-c_{\ell 1}\right)$ are the parametric representation for $0 \leq \lambda_{i \ell 1,2} \leq 1, \ell=1, \ldots, \bar{m}$, and $i=1, \ldots, n$.

The MDT necessitates that we identify the closest point on each of $\ell$, the rectangular plane measured from the centre of $\mathscr{P}_{i}$. We compute the minimum Euclidian distance from the centre of $\mathscr{P}_{i}$ to the surface of the $\ell$ th rectangular plane. The avoidance of the closest point of the surface of the rectangular plane at any time $t \geq 0$ results in the avoidance of the entire plane by $\mathscr{P}_{i}$. Minimising the Euclidean distance between the points $\left(x_{i}, y_{i}, z_{i}\right)$, which is the centre of $\mathscr{P}_{i}$ and the $\ell$ th rectangular plane, yields 


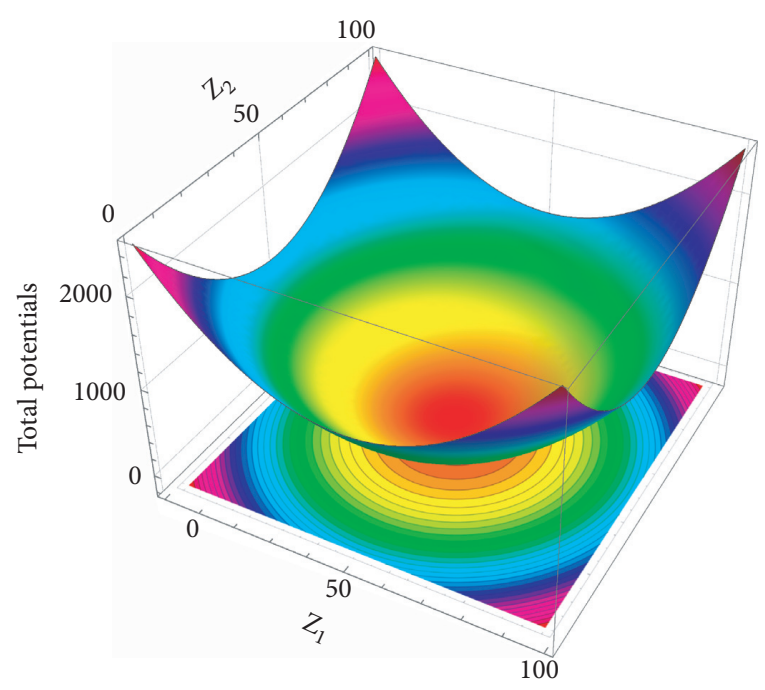

(a)

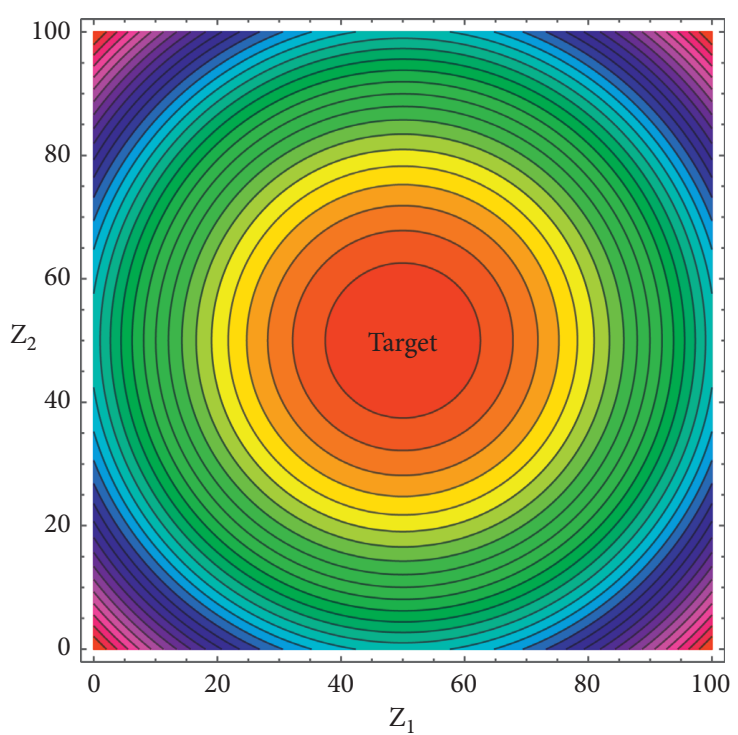

(b)

Figure 4: The 3D visualization of the attractive potential fields and the analogous contour plot (50 contours) generated using the target attractive function, equation (4). (a) 3D visualization. (b) Contour plot.

$$
\begin{aligned}
& \lambda_{i \ell 1}=\left(x_{i}-a_{\ell 1}\right) \bar{c}_{\ell 1}+\left(y_{i}-b_{\ell 1}\right) \bar{d}_{\ell 1}+\left(z_{i}-c_{\ell 1}\right) \bar{h}_{\ell 1}, \\
& \lambda_{i \ell 2}=\left(x_{i}-a_{\ell 1}\right) \bar{c}_{\ell 2}+\left(y_{i}-b_{\ell 1}\right) \bar{d}_{\ell 2}+\left(z_{i}-c_{\ell 1}\right) \bar{h}_{\ell 2},
\end{aligned}
$$

where

$$
\begin{aligned}
& \bar{c}_{\ell 1}=\frac{\left(a_{\ell 2}-a_{\ell 1}\right)}{\left(a_{\ell 2}-a_{\ell 1}\right)^{2}+\left(b_{\ell 2}-b_{\ell 1}\right)^{2}+\left(c_{\ell 2}-c_{\ell 1}\right)^{2}}, \bar{d}_{\ell 1}=\frac{\left(b_{\ell 2}-b_{\ell 1}\right)}{\left(a_{\ell 2}-a_{\ell 1}\right)^{2}+\left(b_{\ell 2}-b_{\ell 1}\right)^{2}+\left(c_{\ell 2}-c_{\ell 1}\right)^{2}}, \\
& \bar{h}_{\ell 1}=\frac{\left(c_{\ell 2}-c_{\ell 1}\right)}{\left(a_{\ell 2}-a_{\ell 1}\right)^{2}+\left(b_{\ell 2}-b_{\ell 1}\right)^{2}+\left(c_{\ell 2}-c_{\ell 1}\right)^{2}}, \bar{c}_{\ell 2}=\frac{\left(a_{\ell 3}-a_{\ell 1}\right)}{\left(a_{\ell 3}-a_{\ell 1}\right)^{2}+\left(b_{\ell 3}-b_{\ell 1}\right)^{2}+\left(c_{\ell 3}-c_{\ell 1}\right)^{2}}, \\
& \bar{d}_{\ell 2}=\frac{\left(b_{\ell 3}-b_{\ell 1}\right)}{\left(a_{\ell 3}-a_{\ell 1}\right)^{2}+\left(b_{\ell 3}-b_{\ell 1}\right)^{2}+\left(c_{\ell 3}-c_{\ell 1}\right)^{2}}, \bar{h}_{\ell 2}=\frac{\left(c_{\ell 3}-c_{\ell 1}\right)}{\left(a_{\ell 3}-a_{\ell 1}\right)^{2}+\left(b_{\ell 3}-b_{\ell 1}\right)^{2}+\left(c_{\ell 3}-c_{\ell 1}\right)^{2}} .
\end{aligned}
$$

The saturation functions are $\lambda_{i \ell 1,2}: \mathbb{R}^{3} \longrightarrow[0,1] \in \mathbb{R}$, defined as

$$
\lambda_{i \ell 1,2}\left(\bar{c}_{\ell 1,2}, \bar{d}_{\ell 1,2}, \bar{h}_{\ell 1,2}\right)=\left\{\begin{array}{l}
0, \quad \text { if } \lambda_{i \ell 1,2}<0 \\
\lambda_{i \ell 1,2}, \quad \text { if } 0 \leq \lambda_{i \ell 1,2} \leq 1, \\
1, \quad \text { if } \lambda_{i \ell 1,2}>1 .
\end{array}\right.
$$

The new obstacle avoidance functions are therefore of the form

$$
\mathrm{RP}_{i \ell}(\mathbf{x})=\frac{1}{2}\left[\left(x_{i}-P x_{i \ell}\right)^{2}+\left(y_{i}-P y_{i \ell}\right)^{2}+\left(z_{i}-P z_{i \ell}\right)^{2}-\left(r p_{i}\right)^{2}\right]
$$

for $i=1, \ldots, n$ and $\ell=1, \ldots, \bar{m}$. The function $\mathrm{RP}_{i \ell}(\mathbf{x})$ is the measure of the distance between the closest point on the surface of the $\ell$ th rectangular-plane-shaped obstacle and the centre of $\mathscr{P}_{i}$.

3.2.3. Moving Obstacles. While in motion, each moving robot itself becomes a moving obstacle to every other mobile robot. For $\mathscr{P}_{i}$ to avoid $\mathscr{P}_{j}$, we consider the following function:

$$
\mathrm{MO}_{i j}(\mathbf{x})=\frac{1}{2}\left[\left(x_{i}-x_{j}\right)^{2}+\left(y_{i}-y_{j}\right)^{2}+\left(z_{i}-z_{j}\right)^{2}-\left(\mathrm{rp}_{i}+\mathrm{rp}_{j}\right)^{2}\right],
$$




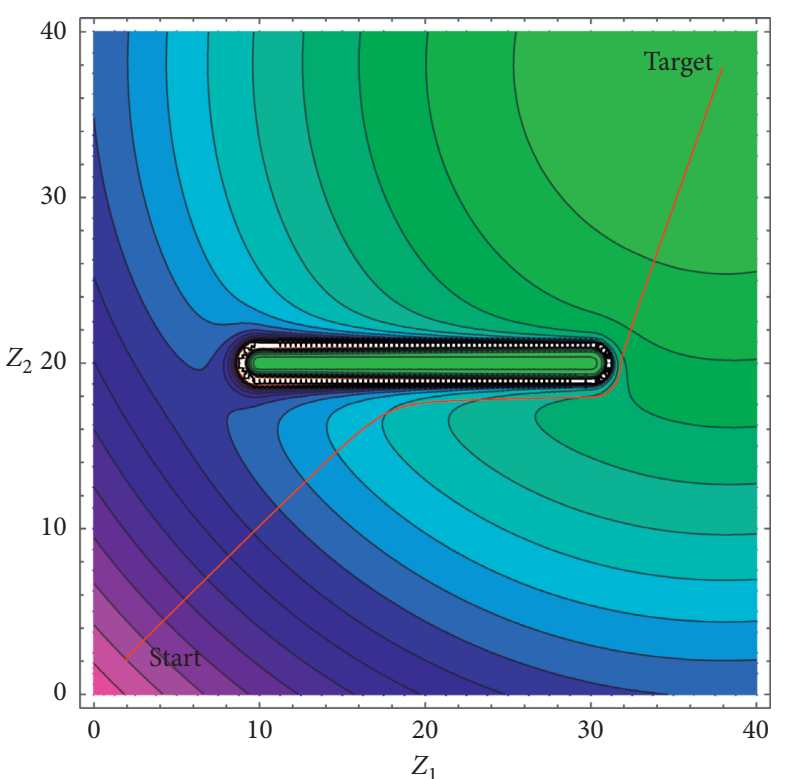

(a)

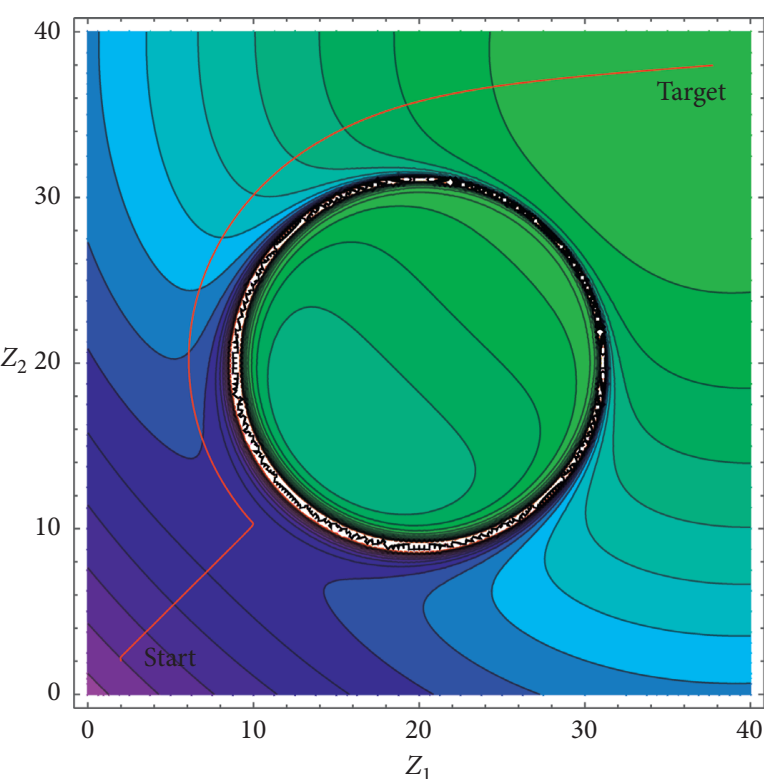

(b)

Figure 5: The contour plots of the total potential fields generated in the presence of a rod-shaped and a disk-shaped obstacle. (a) Rod-shaped obstacle. (b) Disk-shaped obstacle.

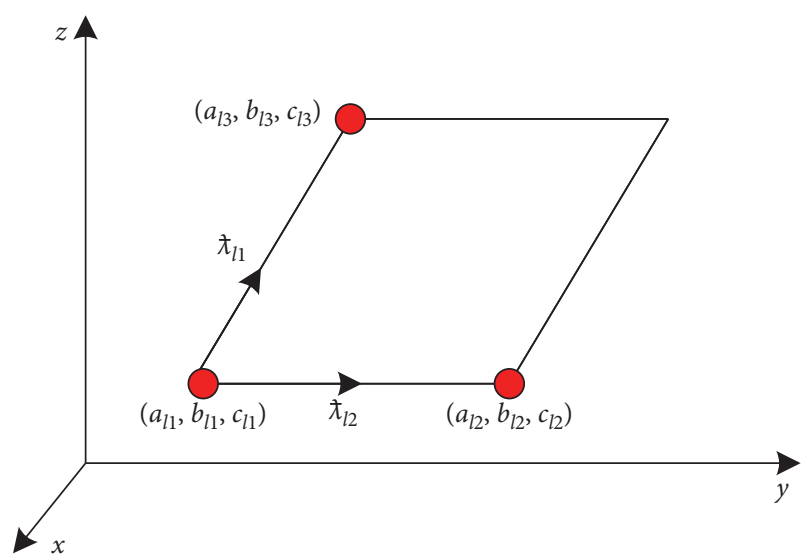

FIgURE 6: Schematic of a rectangular plane.

for $i, j=1, \ldots, n, i \neq j$.

In a nutshell, all these components will now be incorporated to form a Lyapunov function, which will eventually lead to the design of the control inputs for the robotic system.

\section{Design of the Control Inputs}

In this section, we will first construct the Lyapunov function, followed by its time derivative from which we will ultimately extract the nonlinear control inputs for system (2).

4.1. Lyapunov Function. The Lyapunov function, the total potentials that guarantee target convergence and obstacle and collision avoidance, is the sum of the attractive and repulsive potential fields. We begin first by introducing the control/tuning parameters:

(i) $\wp_{i \bar{s}}>0$ and $\bar{s}=1, \ldots, 6$, for the avoidance of the $\bar{s}$ th boundary of the workspace (see Section 3.2.1).

(ii) $\varsigma_{i \ell}>0 \varsigma_{i \ell}>0$ and $\ell=1, \ldots, \bar{m}$, for the avoidance of the surface wall of the $\ell$ th rectangular plane (see Section 3.2.2).

(iii) $\beta_{i j}>0$ and $i, j=1, \ldots, n, i \neq j$, for the avoidance of the $j$ th point-mass mobile robot (see Section 3.2.3).

Suitably combining all the attractive and repulsive potential filed functions using these tuning parameters, we define a Lyapunov function for system (2) as

$$
L(\mathbf{x}):=\sum_{i=1}^{n}\left[V_{i}(\mathbf{x})+G_{i}(\mathbf{x})\left(\sum_{\bar{s}=1}^{6} \frac{\wp_{i \bar{s}}}{\mathrm{WS}_{i \bar{s}}(\mathbf{x})}+\sum_{\ell=1}^{\bar{m}} \frac{\varsigma_{i \ell}}{\mathrm{RP}_{i \ell}(\mathbf{x})}+\sum_{\substack{j=1 \\ j \neq i}}^{n} \frac{\beta_{i j}}{\mathrm{MO}_{i j}(\mathbf{x})}\right)\right],
$$


which is positive over the domain $\mathbb{D}(L)=\left\{\mathbf{x} \in \mathbb{R}^{3 n}: \mathrm{WS}_{i \bar{s}} \quad(\mathbf{x})>0, \bar{s}=1, \ldots, 6, \mathrm{RP}_{i \ell} \quad(\mathbf{x})>0\right.$, $\ell=1, \ldots, \bar{m}$ and $\mathrm{MO}_{i j} \quad(\mathbf{x})>0, j=1, \ldots, n, i \neq j, i=1$, $\ldots, n\}$.
4.2. Control Inputs. Next, we differentiate the various components of $L(\mathbf{x})$ separately with respect to $t$ to obtain (on suppressing $\mathrm{x}$ ) the control inputs for system (2):

$$
\begin{aligned}
& f_{i 1}=\left(1+\sum_{\bar{s}=1}^{6} \frac{\wp_{i \bar{s}}}{\mathrm{WS}_{i \bar{s}}}+\sum_{\ell=1}^{\bar{m}} \frac{\varsigma_{i \ell}}{\mathrm{RP}_{i \ell}}+\sum_{\substack{j=1 \\
j \neq i}}^{n} \frac{\beta_{i j}}{\mathrm{MO}_{i j}}\right)\left(x_{i}-\tau_{i 1}\right)-G_{i} \frac{\wp_{i 1}}{\left(\mathrm{WS}_{i 1}\right)^{2}}+G_{i} \frac{\wp_{i 3}}{\left(\mathrm{WS}_{i 3}\right)^{2}} \\
& -2 G_{i} \sum_{\substack{j=1 \\
j \neq i}}^{n} \frac{\beta_{i j}}{\mathrm{MO}_{i j}}\left(x_{i}-x_{j}\right)-G_{i} \sum_{\ell=1}^{\bar{m}} \frac{\varsigma_{i \ell}}{\mathrm{RP}_{i \ell}}\left(\begin{array}{c}
\left(x_{i}-P x_{i \ell}\right)\left(1-\left(a_{\ell 2}-a_{\ell 1}\right) \bar{c}_{\ell 1}-\left(a_{\ell 3}-a_{\ell 1}\right) \bar{c}_{\ell 2}\right) \\
-\left(y_{i}-P y_{i \ell}\right)\left(\left(b_{\ell 2}-b_{\ell 1}\right) \bar{c}_{\ell 1}+\left(b_{\ell 3}-b_{\ell 1}\right) \bar{c}_{\ell 2}\right) \\
-\left(z_{i}-P z_{i \ell}\right)\left(\left(c_{\ell 2}-c_{k 1}\right) \bar{c}_{\ell 1}+\left(c_{\ell 3}-c_{\ell 1}\right) \bar{c}_{\ell 2}\right)
\end{array}\right), \\
& f_{i 2}=\left(1+\sum_{\bar{s}=1}^{6} \frac{\wp_{i \bar{s}}}{\mathrm{WS}_{i \bar{s}}}+\sum_{\ell=1}^{\bar{m}} \frac{\varsigma_{i \ell}}{\mathrm{RP}_{i \ell}}+\sum_{\substack{j=1 \\
j \neq i}}^{n} \frac{\beta_{i j}}{\mathrm{MO}_{i j}}\right)\left(y_{i}-\tau_{i 2}\right)+G_{i} \frac{\wp_{i 2}}{\left(\mathrm{WS}_{i 2}\right)^{2}}-G_{i} \frac{\wp_{i 4}}{\left(\mathrm{WS}_{i 4}\right)^{2}} \\
& -2 G_{i} \sum_{\substack{j=1 \\
j \neq i}}^{n} \frac{\beta_{i j}}{\mathrm{MO}_{i j}}\left(y_{i}-y_{j}\right)-G_{i} \sum_{\ell=1}^{\bar{m}} \frac{\varsigma_{i \ell}}{\mathrm{RP}_{i \ell}}\left(\begin{array}{c}
-\left(x_{i}-P x_{i \ell}\right)\left(\left(a_{\ell 2}-a_{\ell 1}\right) \bar{d}_{\ell 1}+\left(a_{\ell 3}-a_{\ell 1}\right) \bar{d}_{\ell 2}\right) \\
+\left(y_{i}-P y_{i \ell}\right)\left(1-\left(b_{\ell 2}-b_{\ell 1}\right) \bar{d}_{\ell 1}-\left(b_{\ell 3}-b_{\ell 1}\right) \bar{d}_{\ell 2}\right) \\
-\left(z_{i}-P z_{i \ell}\right)\left(\left(c_{\ell 2}-c_{\ell 1}\right) \bar{d}_{\ell 1}+\left(c_{\ell 3}-c_{\ell 1}\right) \bar{d}_{\ell 2}\right)
\end{array}\right), \\
& f_{i 3}=\left(1+\sum_{\bar{s}=1}^{6} \frac{\wp_{i \bar{s}}}{\mathrm{WS}_{i \bar{s}}}+\sum_{\ell=1}^{\bar{m}} \frac{\varsigma_{i \ell}}{\mathrm{RP}_{i \ell}}+\sum_{\substack{j=1 \\
j \neq i}}^{n} \frac{\beta_{i j}}{\mathrm{MO}_{i j}}\right)\left(z_{i}-\tau_{i 3}\right)-G_{i} \frac{\wp_{i 5}}{\left(\mathrm{WS}_{i 5}\right)^{2}}+G_{i} \frac{\wp_{i 6}}{\left(\mathrm{WS}_{i 6}\right)^{2}} \\
& -2 G_{i} \sum_{\substack{j=1 \\
j \neq i}}^{n} \frac{\beta_{i j}}{\mathrm{MO}_{i j}}\left(z_{i}-z_{j}\right)-G_{i} \sum_{\ell=1}^{\bar{m}} \frac{\varsigma_{i \ell}}{\mathrm{RP}_{i \ell}}\left(\begin{array}{c}
-\left(x_{i}-P x_{i \ell}\right)\left(\left(a_{\ell 2}-a_{\ell 1}\right) \bar{h}_{\ell 1}+\left(a_{\ell 3}-a_{\ell 1}\right) \bar{h}_{\ell 2}\right) \\
-\left(y_{i}-P y_{i \ell}\right)\left(\left(b_{\ell 2}-b_{\ell 1}\right) \bar{h}_{\ell 1}+\left(b_{\ell 3}-b_{\ell 1}\right) \bar{h}_{\ell 2}\right) \\
+\left(z_{i}-P z_{i \ell}\right)\left(1-\left(c_{\ell 2}-c_{\ell 1}\right) \bar{h}_{\ell 1}-\left(c_{\ell 3}-c_{\ell 1}\right) \bar{h}_{\ell 2}\right)
\end{array}\right) \text {, }
\end{aligned}
$$

for $i=1, \ldots, n$. In order to ensure stability in the sense of Lyapunov of system (2), we define the accompanying continuous velocity control laws as follows:

$$
\begin{aligned}
& v_{i}=-\frac{1}{\alpha_{i 1}} f_{i 1}, \\
& w_{i}=-\frac{1}{\alpha_{i 2}} f_{i 2}, \\
& u_{i}=-\frac{1}{\alpha_{i 3}} f_{i 3},
\end{aligned}
$$

for $i=1, \ldots, n$. Our main theorem, given next, uses these laws to prove the stability of our system.

\section{Stability Analysis}

Using the notations $\mathbf{x}_{e_{i}}:=\left(\tau_{i 1}, \tau_{i 2}, \tau_{i 3}\right) \in \mathbb{R}^{3}$ and $\mathbf{x}_{e}:=\left(\mathbf{x}_{e_{1}}, \ldots, \mathbf{x}_{e_{n}}\right) \in \mathbb{R}^{3 n}$, we state the following theorem.

Theorem 1. A stable equilibrium point of system (2) is $\mathbf{x}_{e} \in \mathbb{D}(L(\mathbf{x}))$. 
Proof. Since the Lyapunov function $L(\mathbf{x})$ of system (2) is defined, continuous and positive over the domain $\mathbb{D}(L)=$ $\left\{\mathbf{x} \in \mathbb{R}^{3 n}: \mathrm{WS}_{i \bar{s}}(\mathbf{x})>0, \bar{s}=1, \ldots, 6, \mathrm{RP}_{i \ell}(\mathbf{x}), \ell=1, \ldots, \bar{m}\right.$

and $\left.\mathrm{MO}_{i j}(\mathbf{x})>0, j=1, \ldots, n, i \neq j, i=1, \ldots, n\right\}$, it can easily be verified that $L(\mathbf{x})$ satisfies the following properties:

(1) $L(\mathbf{x})$ is continuous in the region $\mathbb{D}$ in the neighborhood of the point $\mathbf{x}_{e}$ of system (2)

(2) $L\left(\mathbf{x}_{e}\right)=0$

(3) $L(\mathbf{x})>0, \forall \mathbf{x} \in \mathbb{D}\left((L(\mathbf{x})) / \mathbf{x}_{e}\right)$

Then, along a solution of system (2), we have

$$
\dot{L}_{(1)}(\mathbf{x})=\sum_{i=1}^{n}\left[f_{i 1} \dot{x}_{i}+f_{i 2} \dot{y}_{i}+f_{i 3} \dot{z}_{i}\right] .
$$

Using (17), we have the following time derivative of $L(\mathbf{x})$ seminegative definite function for system (2):

$$
\dot{L}_{(1)}(\mathbf{x})=-\sum_{i=1}^{n}\left[\alpha_{i 1} v_{i}^{2}+\alpha_{i 2} w_{i}^{2}+\alpha_{i 3} u_{i}^{2}\right] \leq 0 \text {. }
$$

Therefore, $\dot{L}_{(1)}(\mathbf{x}) \leq 0, \forall \mathbf{x} \in \mathbb{D}(L(\mathbf{x}))$ and $\dot{L}_{(1)}\left(\mathbf{x}_{e}\right)=0$. Moreover $L(\mathbf{x}) \in C^{1}(\mathbb{D}(L(\mathbf{x})))$; hence, for system $(2), L(\mathbf{x})$ is classified as its Lyapunov function and $\mathbf{x}_{e}$ is a stable equilibrium point.

Furthermore, with the design of the new controllers and the stability analysed for the robotic system, the effectiveness of the control scheme is verified using computer simulations.

\section{Simulation Results}

The three situations given in this section capture realistic situations to illustrate the adequacy, effectiveness, and robustness of the velocity-based controllers and the control scheme. In the following scenarios, the data use international units in the sense that parameters are unitless whereas the times can be treated consistently. For instance, the units of time can follow the international units like seconds or minutes and the distance can be in centimeters or meters.

6.1. Scenario 1. In this scenario, we consider a simple setup where $\mathscr{P}_{i}$ navigates itself from its initial position to its predefined target in the presence of a fixed rectangularplane obstacle. There are 3 point-mass mobile robots and a rectangular-plane obstacle. Each of the point-mass mobile robots avoids each other as well as the rectangular-plane obstacle while en route to its target. It is very interesting to observe the proximity of the point-mass mobile robots to the wall as it tries to evade it, exerting just enough energy to move above the wall and converge to its targets. The behavior exhibited by $\mathscr{P}_{i}$ is quite intriguing as it mimics a similar behavior that a swarm of birds exhibits while the swarm approaches a wall. Figure 7(a) shows the default 3D view and Figure $7(\mathrm{~b})$ shows the top $3 \mathrm{D}$ view while Figure 7 (c) shows the front $3 \mathrm{D}$ view of the motion of the point-mass mobile robots. The obstacle has transparency to allow us to view the position and path of $\mathscr{P}_{i}$. The blue sphere represents the motion of $\mathscr{P}_{i}$ at $t=0$ unit of time, red sphere at $t=700$ units of time, green sphere at $t=$ 3500 units of time, and purple sphere at $t=15000$ units of time. Table 1 provides all the values of the initial conditions, constraints, and different parameters utilised in the simulation.

6.2. Scenario 2. Here we model rectangular towers, which could represent tall buildings in cities. These towers, constructed with 15 planes, block the path of a swarm of 5 pointmass mobile robots. The agents are observed to start from their initial positions and maneuver themselves to their predefined targets, while ensuring avoidance of the towers as well as interindividual collision avoidance. Each $\mathscr{P}_{i}$ computes the shortest and a collision-free path to its destination. Split maneuvers are observed while the robots are en route along their paths. Such an example with multiple towers can be used to model the obstacle avoidance capability of UAVs. Figure 8(a) shows the default $3 \mathrm{D}$ view and Figure $8(\mathrm{~b})$ shows the top 3D view, while Figure 8(c) shows the front $3 \mathrm{D}$ view of the motion of the point-mass mobile robots. The blue sphere represents the motion of $\mathscr{P}_{i}$ at $t=0$ unit of time, red sphere at $t=700$ units of time, green sphere at $t=3120$ units of time, and purple sphere at $t=8000$ units of time. Table 2 provides all the values of the initial conditions, constraints, and different parameters utilised in the simulation, if different from the previous scenario. For the construction of the towers, the reader is referred to the figures for the extraction of the coordinates.

6.3. Scenario 3. An interesting research domain involves tunnel passing maneuvers. In this scenario, there are 3 point-mass mobile robots and we design tunnels using rectangular planes. We use 8 rectangular planes to construct the tunnel. In addition, the top and one of the side views have been strategically made transparent to show the trajectory and the position of $\mathscr{P}_{i}$ as it maneuvers through the tunnel. The snapshots show the way the pointmass robots strategize their motion to allow which robot will pass through the tunnel first and how they will converge to their respective predefined targets. Drones could be deployed in areas that are deemed to be "dull, dirty, and dangerous" as well as "difficult" such as that of collapsed tunnel passages to capture, store, check, and send data for analysis. Figure 8(a) shows the default 3D view and Figure 8(b) shows the top 3D view while Figure 8(c) shows the front 3D view of the motion of the point-mass mobile robots. The blue sphere represents the motion of $\mathscr{P}_{i}$ at $t=0$ unit of time, red sphere at $t=550$ 


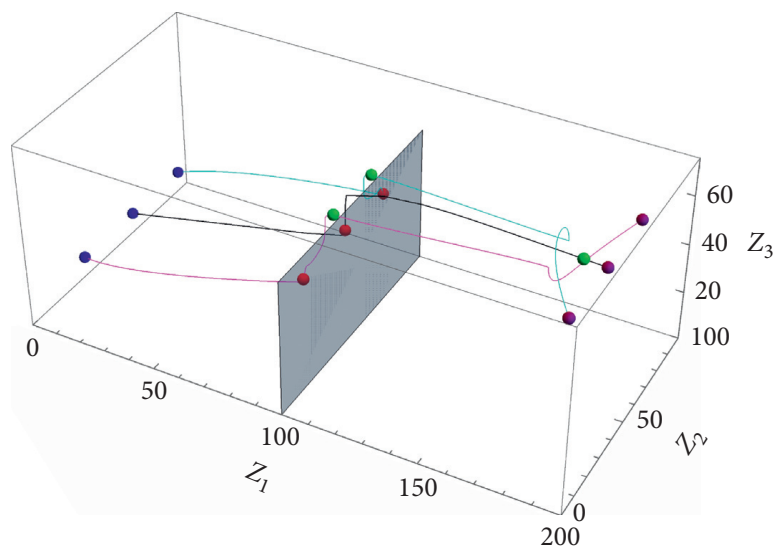

(a)

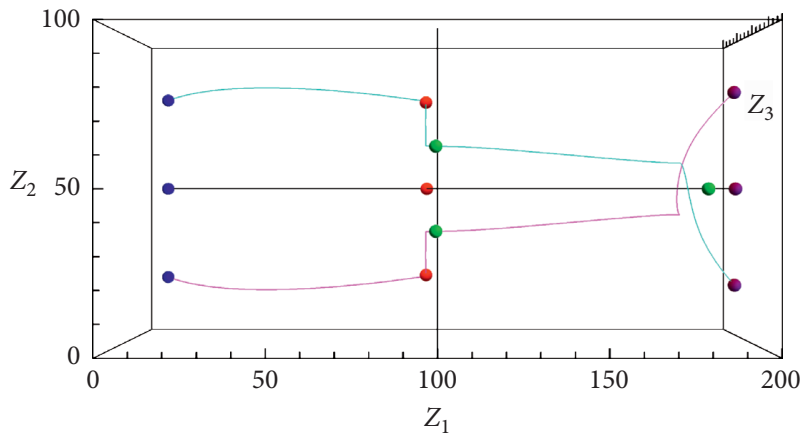

(b)

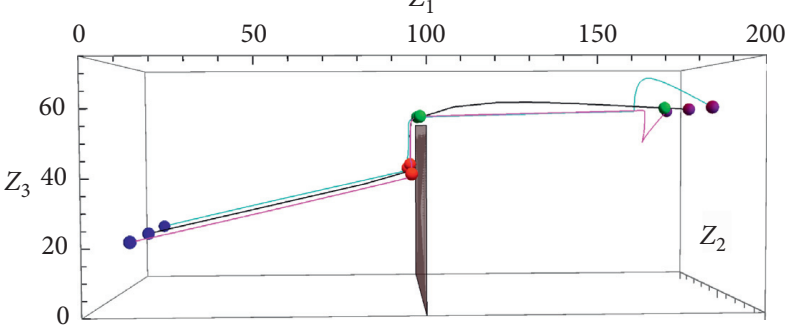

(c)

Figure 7: Scenario 1. The different viewpoints of $\mathscr{P}_{i}$ while it is in motion to its target with one rectangular-plane obstacle. (a) Default 3D motion view. (b) Top 3D motion view. (c) Front 3D motion view.

TABLE 1: Scenario 1 . The parameters utilised in the numerical simulation. There are 3 point-mass mobile robots $(n=3)$ and 1 rectangularplane obstacle $(\bar{m}=t 1)$.

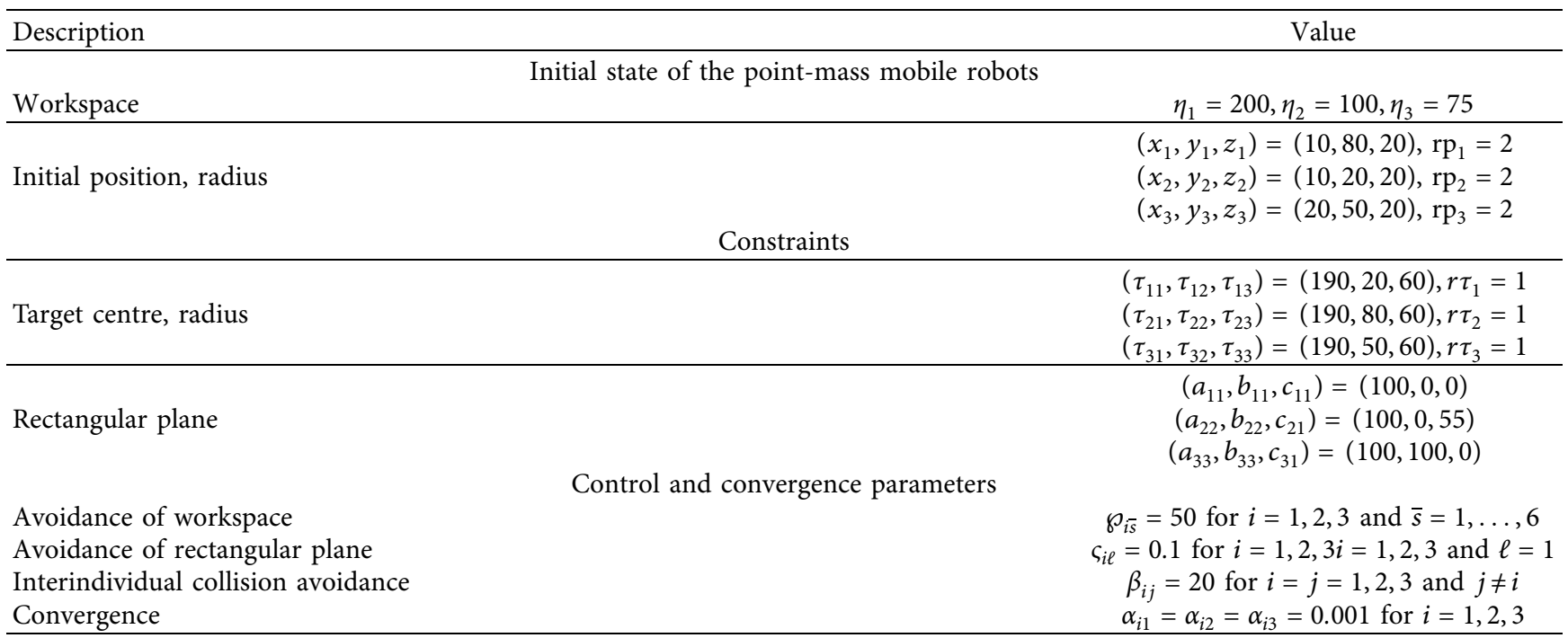

units of time, green sphere at $t=2110$ units of time, and purple sphere at $t=8000$ units of time. Table 3 provides all the values of the initial conditions, constraints, and different parameters utilised in the simulation, if different from the previous scenario. The evolution of the Lyapunov function and its time derivative together with its control inputs along the trajectories are depicted in Figure 9, respectively. 


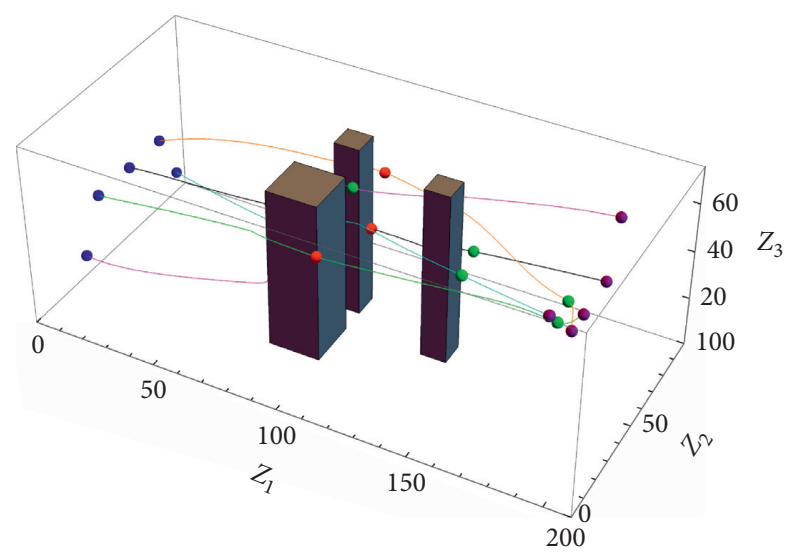

(a)

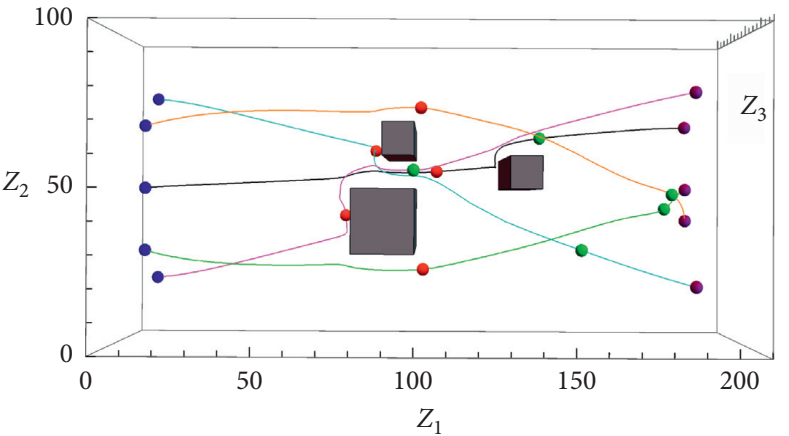

(b)

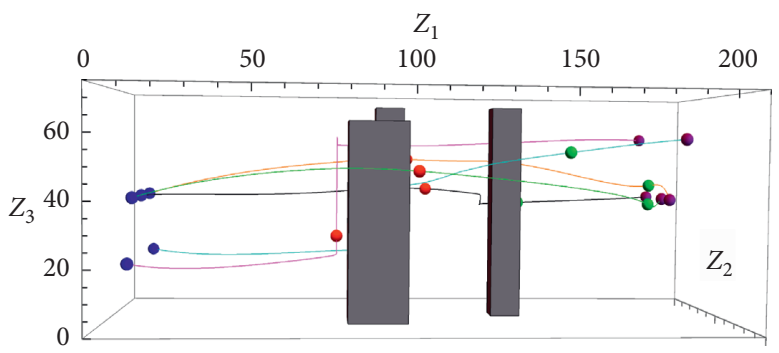

(c)

Figure 8: Scenario 2. The different viewpoints of $\mathscr{P}_{i}$ while it is in motion to its target with towers constructed from the rectangular planes. (a) Default 3D motion view. (b) Top 3D motion view. (c) Front 3D motion view.

TABLE 2: Scenario 2. The parameters utilised in the numerical simulation, with $n=3$ and $\bar{m}=15$.

\begin{tabular}{|c|c|}
\hline Description & Value \\
\hline \multicolumn{2}{|c|}{ Initial state of the point-mass mobile robots } \\
\hline \multicolumn{2}{|r|}{$\begin{array}{l}\left(x_{3}, y_{3}, z_{3}\right)=(10,50,40), \mathrm{rp}_{3}=2 \\
\left(x_{4}, y_{4}, z_{4}\right)=(10,70,40), \mathrm{rp}_{3}=2 \\
\left(\left(x_{5}, y_{5}, z_{5}\right)=(10,30,40), \mathrm{rp}_{3}=2\right.\end{array}$} \\
\hline \multicolumn{2}{|c|}{$\begin{array}{ll}\left(\tau_{31}, \tau_{32}, \tau_{33}\right)=(190,70,40), r \tau_{3}=1 \\
& \left(\tau_{41}, \tau_{42}, \tau_{43}\right)=(190,40,40), r \tau_{3}=1 \\
& \left(\tau_{51}, \tau_{52}, \tau_{53}\right)=(190,50,40), r \tau_{3}=1\end{array}$} \\
\hline $\begin{array}{l}\text { Avoidance of workspace } \\
\text { Avoidance of rectangular plane } \\
\text { Interindividual collision avoidance }\end{array}$ & $\begin{array}{c}\wp_{i \bar{s}}=20 \text { for } i=1, \ldots, 5 i=1, \ldots, 5 \text { and } \bar{s}=1, \ldots, 6 \\
\varsigma_{i \ell}=0.5, \text { for } i=1, \ldots, 5 \text { and } \ell=1, \ldots, 15 \\
\beta_{i j}=5 \text { for } i=j=1, \ldots, 5 \text { and } j \neq i\end{array}$ \\
\hline
\end{tabular}

TABLe 3: Scenario 3. The parameters utilised in the numerical simulation, with $n=3$ and $\bar{m}=8$.

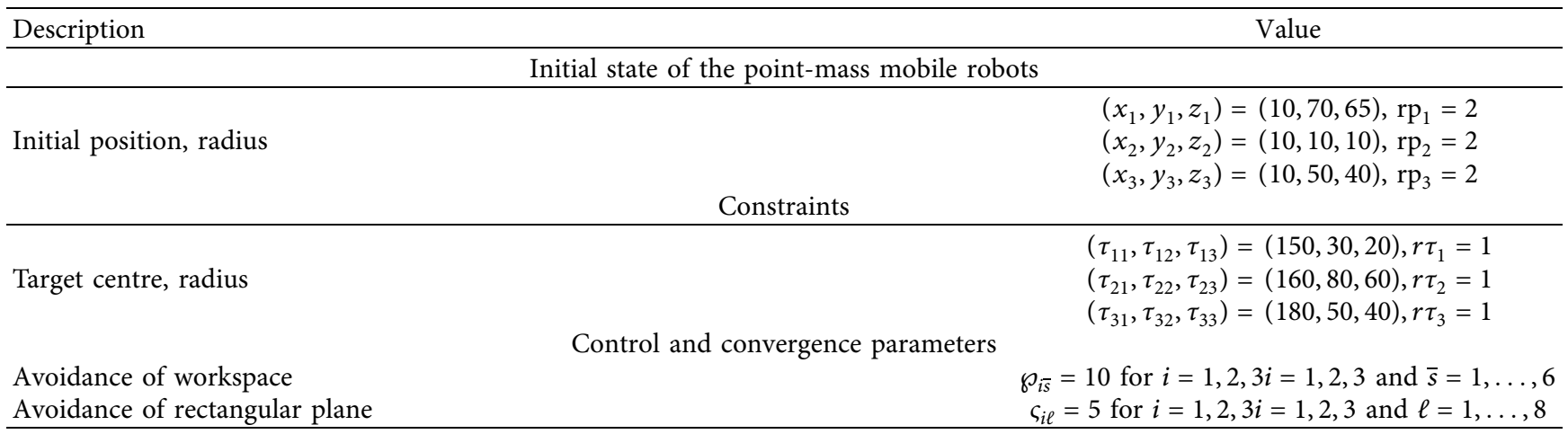



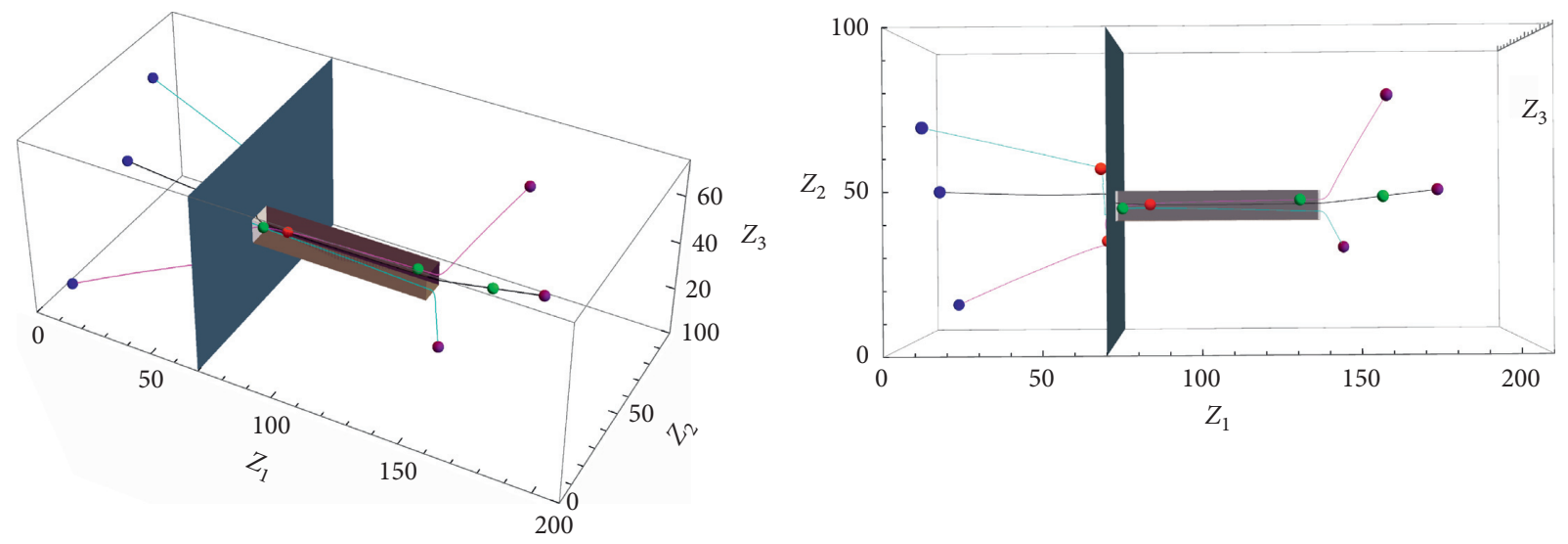

(a)

(b)

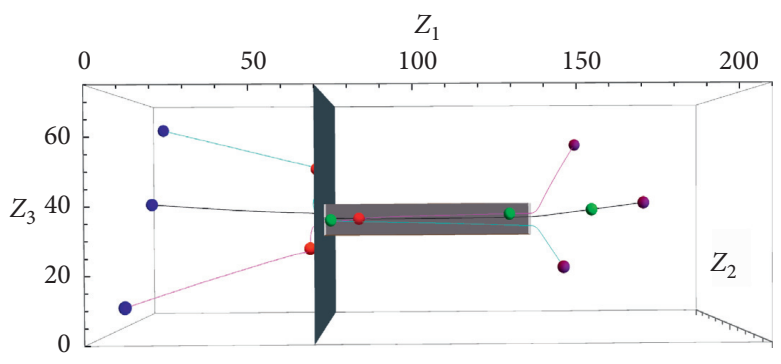

(c)

Figure 9: Scenario 3. The evolutionary behavior of the Lyapunov function, its time derivative, and its control inputs along the trajectories of Figure 10. (a) Lyapunov function. (b) Lyapunov function time derivative. (c) Controllers.

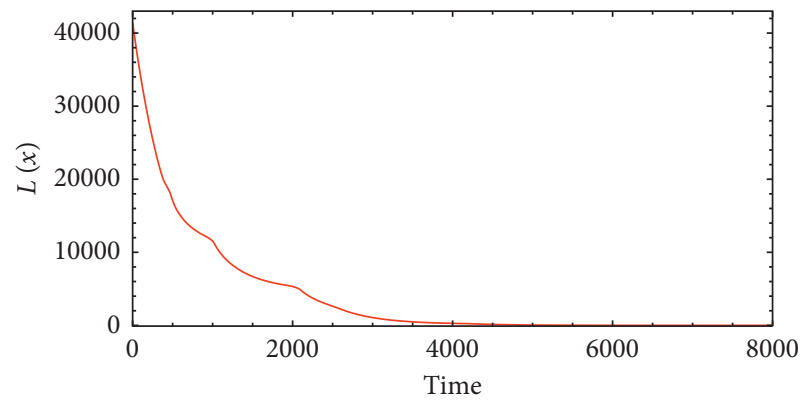

(a)

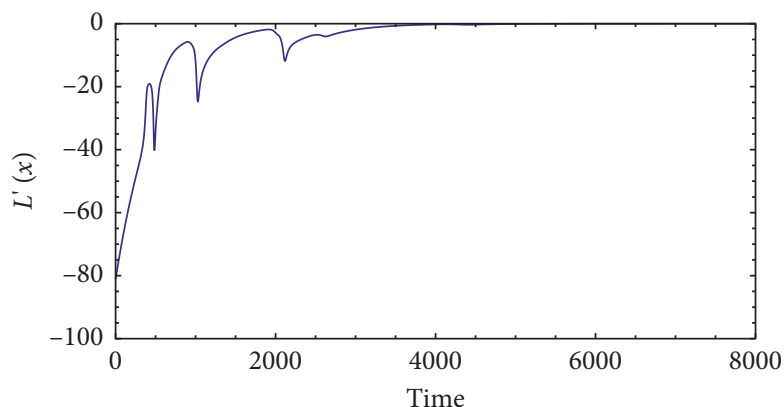

(b)

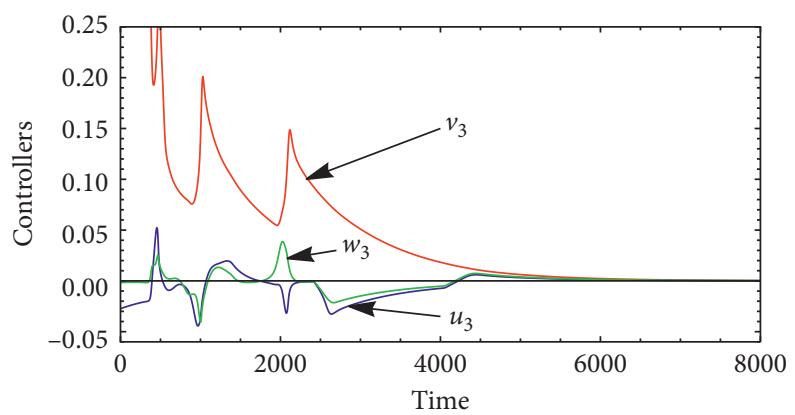

(c)

Figure 10: Scenario 3. The different viewpoints of $\mathscr{P}_{i}$ while it is in motion to its target with tunnel passing maneuvers. (a) Default 3D motion view. (b) Top 3D motion view. (c) Front 3D motion view. 
Interestingly, the behavior exhibited in this scenario can be seen in nature, namely, the leader-follower strategy, where the leader guides the followers to food sources, safety, and so on. We note that the leader-follower strategy, cooperative hunting, and avoidance in the military are dronebased applications considered common nowadays.

\section{Conclusions and Future Work}

Mathematical modelling and the design of motion planners for robotic systems are a complex, computationally expensive yet a fascinating research area. In this paper, the LbCS was applied to derive a set of robust, unique continuous time-invariant velocity-based control inputs that effectively handle the problem of MPC of point-mass mobile robots in a dynamic environment that, for the absolute first time, incorporates rectangular-plane obstacles. The convergence of the mobile robots to a neighborhood of a predefined target is ensured by the Lyapunov direct method. The effectiveness and robustness of the control scheme were illustrated via computer simulation of virtual scenarios that depicts real-life situations.

To the authors' knowledge, this is the first time in literature whereby the MDT was used to derive the mathematical functions for the successful avoidance of rectangular-plane-shaped obstacles. The introduction of the rectangular-plane obstacle into the MPC problem has created new dimensions and potentials for research. The advantages of the MDT are numerous such as making it possible for plane-shaped (and other irregular) obstacles to be treated within the motion planners, help in simplifying collision-avoidance algorithms, and permit maximum freespace for the robots traversing the workspace.

This work paves the way for numerous future directions. Our principal objective is to extend the rectangular-plane obstacles in a workspace for the MPC of a flock of quadrotors performing hovering maneuvers and undergoing split-and-rejoin maneuvers when encountered with towers and tunnels.

\section{Data Availability}

The research data for this article are purely simulationbased. These would be available upon request.

\section{Conflicts of Interest}

The authors declare that there are no conflicts of interest regarding the publication of this paper.

\section{References}

[1] K. Molloy, D. Laurent, M. Vaisset, T. Siméon, and Juan Cortés, "Simultaneous system design and path planning: a sampling-based algorithm," The International Journal of Robotics Research, vol. 38, no. 2-3, pp. 375-387, 2019.

[2] A. Gasparetto, P. Boscariol, A. Lanzutti, and R. Vidoni, Path Planning and Trajectory Planning Algorithms: A General Overview, Springer International Publishing, Berlin, Germany, 2015.
[3] N. Lashkari, M. Biglarbegian, S. X. Yang, and Yang, "Development of novel motion planning and controls for a series of physically connected robots in dynamic environments," Journal of Intelligent \& Robotic Systems, vol. 95, no. 2, pp. 291-310, 2019.

[4] B. N. Sharma, J. Raj, and J. Vanualailai, "Navigation of carlike robots in an extended dynamic environment with swarm avoidance," International Journal of Robust and Nonlinear Control, vol. 28, no. 2, pp. 678-698, 2018.

[5] M. Liu, H. Ma, J. Li, and S. Koenig, "Task and path planning for multi-agent pickup and delivery," in Proceedings of the 18th International Conference on Autonomous Agents and MultiAgent Systems, AAMAS '19, Richland, SC, USA, May 2019.

[6] D. Ali and V. Sezer, "Motion planning and control with randomized payloads using deep reinforcement learning," in Proceedings of the 2019 Third IEEE International Conference on Robotic Computing (IRC), Naples, Italy, February 2019.

[7] J. Raj, K. Raghuwaiya, and J. Vanualailai, "Novel lyapunovbased autonomous controllers for quadrotors," IEEE Access, vol. 8, no. 1, pp. 47393-47406, 2020.

[8] F. Ho, A. Salta, R. Geraldes, A. Goncalves, M. Cavazza, and H. Prendinger, "Multi-agent path finding for uav traffic management," in Proceedings of the 18th International Conference on Autonomous Agents and MultiAgent Systems, AAMAS '19, Richland, SC, USA, May 2019.

[9] J. T. Ebert, M. Gauci, and R. Nagpal, "Multi-feature collective decision making in robot swarms," in Proceedings Of the 17th International Conference On Autonomous Agents and MultiAgent Systems, AAMAS '18, Richland, SC, USA, May 2018.

[10] B. Sharma, J. Vanualailai, and S. Singh, "Motion planning and posture control of multiple n-link doubly nonholonomic manipulators," Robotica, vol. 35, no. 1, pp. 1-25, 2017.

[11] D. A. L. Burbano, P. Delellis, and M. dibernardo, "Self-tuning proportional integral control for consensus in heterogeneous multi-agent systems," European Journal of Applied Mathematics, vol. 27, no. 6, pp. 923-940, 2016.

[12] J. Raj, R. Krishna, S. Singh, B. Sharma, and J. Vanualailai, N. Che, "Swarming intelligence of 1-trailer systems," Hamzah Asyrani Sulaiman, Mohd Azlishah Othman, Mohd Fairuz Iskandar Othman, Yahaya Abd Rahim, and, pp. 251-264, Springer International Publishing, Berlin, Germany, 2016.

[13] R. Krishna, B. Sharma, and J. Vanualailai, "Leader-follower based locally rigid formation control," Journal of Advanced Transportation, vol. 2018, Article ID 5278565, 14 pages, 2018.

[14] J. Raj, R. Krishna, J. Vanualailai, and B. Sharma, "Navigation of car-like robots in three-dimensional space," in Proceedings of the 2018 5th Asia-Pacific World Congress on Computer Science and Engineering (APWC on CSE), Nadi, Fiji, December 2018.

[15] T. S. A. Ebtehal, "A complete multi-robot path-planning algorithm: jaamas track," in Proceedings of the 18th International Conference on Autonomous Agents and MultiAgent Systems, AAMAS '19, Richland, SC, USA, May 2019.

[16] B. Sharma, J. Vanualailai, and A. Prasad, "A d $\phi$-strategy: facilitating dual-formation control of a virtually connected team $\mathrm{d} \phi$ - strategy: facilitating dual-formation control of a virtually connected team," Journal of Advanced Transportation, vol. 2017, Article ID 9213805, 17 pages, 2017.

[17] R. Krishna and S. Singh, "Formation types of multiple steerable 1-trailer mobile robots via split/rejoin maneuvers," New Zealand Journal of Mathematics, vol. 43, no. 7-21, 2013.

[18] J. Vanualailai, "Stable emergent formations for a swarm of autonomous car-like vehicles," International Journal of Advanced Robotic Systems, vol. 16, no. 5, pp. 1-17, 2019. 
[19] D. Albani, T. Manoni, D. Nardi, and V. Trianni, "Dynamic uav swarm deployment for non-uniform coverage," in Proceedings of the 17th International Conference on Autonomous Agents and MultiAgent Systems, AAMAS '18, Richland, SC, USA, May 2018.

[20] E. Hartuv, N. Agmon, and S. Kraus, "Scheduling spare drones for persistent task performance under energy constraints," in Proceedings Of the 17th International Conference On Autonomous Agents and MultiAgent Systems, AAMAS '18, Richland, SC, USA, May 2018.

[21] B. Sharma, J. Vanualailai, and S. Singh, "Tunnel passing maneuvers of prescribed formations," International Journal of Robust and Nonlinear Control, vol. 24, no. 5, pp. 876-901, 2014.

[22] M. d. S. Arantes, C. F. M. Toledo, B. C. Williams, and M. Ono, "Collision-free encoding for chance-constrained nonconvex path planning," IEEE Transactions on Robotics, vol. 35, no. 2, pp. 433-448, 2019.

[23] K. Ariu, F. Cheng, M. . da Silva Arantes, C. Toledo, and B. C. Williams, "Chance-constrained path planning with continuous time safety guarantees," in Proceedings of the Workshops at the Thirty-First AAAI Conference on Artificial Intelligence, Palo Alto, CA, USA, February 2017. 